\title{
CRYSTALS: USAGE AS MEMORY DEVICES
}

\author{
B. Prasanthi ${ }^{1}$, P. Abhilash ${ }^{2}$, G. Raju ${ }^{3}$ \\ ${ }^{1}$ Senior Assistant Prof. CSE, MGIT, Hyderabad \\ ${ }^{2}$ Student, MGIT, Hyderabad \\ ${ }^{3}$ Student, MGIT, Hyderabad
}

\begin{abstract}
Memory has become an integral part of daily life. Many devices have emerged from years. This paper gives a brief idea about how crystals can be used for storing bits of data, as an alternative for traditional transistors and gates. It suggests a novel way of storing data in crystals suspended in an optically sensitive gel, creating a whole new generation of non-solid memories. The advantages of memory using crystals when compared to traditional memories and methods to construct the same have been highlighted.
\end{abstract}

Keywords: transistors, gates, crystals

\section{INTRODUCTION}

Nowadays the very first thing that comes to our mind when we think of any digital device is memory. This memory of a digital device is in the form of 'ones' and 'zeroes ' called bits. These bits are stored using many transistors and gates. The memory density of a digital device is directly proportional to number of transistors and gates used in it. Gordon Moore stated that the number of transistors in an electronic device doubles approximately for every two years. According to this law we might hit a technology brick wall because we already have very huge number of transistors and gates per unit area and further increase in these silicon devices per unit area will not only increase manufacturing costs but also increases heat generated by these devices. "It is true that simply taking old processor architectures and scaling them won't work anymore," said William J. Dally, chief scientist at Nvidia [1]. This shows the urgency of the situation to have an alternative to these silicon based devices. This paper suggests some views and ideas about several crystal properties that can be used to store bits.

\section{MATERIALS REQUIRED FOR}

\section{CONSTRUCTION}

A crystal or crystalline solid is a solid material whose constituent atoms, molecules or ions are arranged in ordered pattern extending in all three spatial dimensions. They show very interesting properties when subjected to heat, light, pressure etc. These properties can be exploited to our use for constructing different memories. Depending upon the method of construction and the property of a crystal that is used, we can have different crystals each exhibiting a particular property. These crystals are to be suspended in gel like substance which is in semi-solid state and whose properties again depend on method used for constructing memory.

Additional hardware might be required for reading, writing and erasing these memories. To summarize, we need crystals of a particular elements or compounds and a gel, to hold these crystals in position.

\section{OPTICAL PROPERTIES OF CRYSTALS} AND SPECTROSCOPY

Certain crystals upon exposure to light show peculiar properties. For example when a quartz crystal or any silver halide crystal is exposed to light it shows a change in its color hence they are called photo-sensitive crystals. This property of photo-sensitivity can be used to store memory. For sake of illustration let's take a silver halide crystal silver chloride. The crystals of silver chloride $(\mathrm{Agcl})$ are suspended in gelatin in order to hold them intact. It also acts as a transparent medium allowing the light to pass through it.

Arrangement of Agcl Crystals in Gelatin: The arrangement of the crystals should be done in such a way that each crystal must be able to face the incident light ray. It can be done by arranging the whole gelatin along with crystals in solid 3-dimensional objects like cylinder, cube etc. where crystals are placed on the surface as well as inside the solid shape in such a way that all of them are exposed to light. This entire setup is enclosed with a skin or cover which is transparent. This provides the mechanical strength to the setup and retains shape.

Write Operation: The write operation is performed by exposing the selected crystals to light. Every crystal that is exposed to light will show a change in color. In this example crystals that are exposed to light will turn bright. These crystals are considered to be storing bit ' 1 ', The remaining crystals which are not exposed to light are considered to be storing bit ' 0 ' . Usually light radiation of high intensity is used to perform write operations. This method is similar to writing data to an optical disk with the help of laser light. The light generated must be focused on selective crystals only. This is similar to focusing light on selected sectors and tracks on an optical disk. Hence existing technology with some minor changes might be used to do this operation. 
Read Operation: This operation is performed by exposing the crystals to a very low intensity light radiation. This light when incident on brighter crystals i.e. on the crystals which were previously written, the reflected light ray will be bright enough indicating the crystal contains bit ' 1 '. On the other hand if this light is incident on the darker crystals it gets absorbed by the darker crystals and there will be no reflected ray indicating that the crystal contains bit ' 0 '. So by focusing this low intensity radiation onto the crystals and studying the pattern of the reflected rays we can perform read operation. This is also similar to reading an optical disk using laser light. Hence existing technology can be used for this purpose also.

Erase Operation: This operation is performed by passing a small electric impulse through the gel that holds the crystals (in our case gelatin) in order to restore the crystals to their original state i.e. zero state. In our case the silver $(\mathrm{Ag})$ which is present as bright precipitate i.e. indicating bit value ' 1 ' returns to its original state i.e. silver halide state $(\mathrm{Agcl})$ indicating bit value ' 0 ' . So with use of small electric potential we can erase these crystals.

\section{Advantages}

The above mentioned approach improves the memory density by increasing the number of crystals per unit length.

It also reduces the usage of electricity since we use electricity to erase the memory only.

It enhances the speed of read, write and erase operations.

It also increases the life time of the memory device since crystals have longer life times when compared to other electronic devices.

It provides the scope of having flexible memory devices in the future instead of having rigid memory devices.

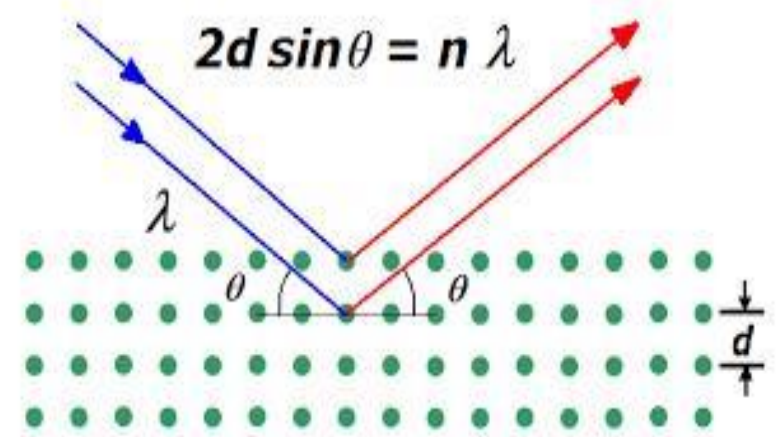

\section{Bragg's Equation}

The above fig shows bragg's equation which stands for $\mathrm{n} \Lambda=2 \mathrm{~d}^{*} \sin \Theta$. This equation shows that how light rays can be used to calculate the seperation between two layers of a crystal. Using the same analogy we can say that it is possible to perform the read, write and erase operations on the crystal as mentioned above by using the light rays of different intensity.

\section{Feasibility of the Suggested Approach}

In this approach we came to notice that the crystals used must be selected with extreme caution. The selected crystals must have certain properties like photo sensitivity, Stability, mechanical strength, uniformity etc. This debates the concept of selecting appropriate crystal for the use.

Same argument applies for the gel that is used to hold the crystals.

Also the arrangement of these crystals in the gel as specified is difficult.

The dimensions of these crystals must be very small so as to increase the memory density.

The use of light radiation of different intensities is difficult but it is not impossible to achieve.

\section{PIEZOELECTRICITY}

Piezoelectricity is the electric charge that accumulates in certain solid materials like crystals in response to applied mechanical stress. This peculiar property of certain crystals can be exploited to our advantage. As an example lead zirconate titanate crystal show piezoelectric effect when subject to pressure which deforms it by about $0.1 \%$ of its original dimension. So these crystals may be suspended in a gel which is in a semi solid state so as to hold them together.

Arrangement of PZT (lead zirconate titanate) Crystals in gel: The arrangement of the crystals should be done in such a way that each crystal must be able to take the same amount of mechanical strength applied. It can be done by arranging the whole gel along with crystals in solid 3dimensional objects like cylinder, cube etc. where crystals are placed on the surface as well as inside the solid shape in such a way that all are symmetrically located with respect to each other. This entire setup is enclosed with a skin or cover which is hard and strong. This provides the mechanical strength to the setup and retains shape.

Write Operation: The write operation is performed by exposing the selected crystals to mechanical stress. Every crystal that is exposed to pressure will develop an E.M.F in it. This charge is stored in it for a considerable amount of time because the gel acts as an insulator allowing the crystals to retain their charge. The mechanical stress is generated by applying pressure on the selected crystals by electrodes which also act as E.M.F sensors for read operation. Thus the crystal which is subjected to stress is considered to be storing ' 1 ' and the rest of the crystals are considered to be storing ' 0 '.

Read Operation: This operation is performed by reading the E.M.F values in the crystals by voltage sensor. This voltage sensor reads bit ' 1 ' when there is E.M.F in the crystal or else it reads bit ' 0 '. 
Erase Operation: This operation is performed by sending voltage impulses to the crystals which previously contain bit value ' 1 ' i.e. they are initially subjected to pressure. These voltages are sent through the same voltage sensors that are attached to the crystals. Since piezoelectricity is a reversible effect the crystals return to their original shape and the stored voltage in them is then removed by the same electrodes or voltage sensors. This restores the crystals to their original state and also with no charge in them.

\section{Feasibility of the Suggested Approach:}

In this approach we came to notice that the crystals used must be selected with extreme caution.

The selected crystals must have certain properties like piezoelectricity, considerable rigidity and flexibility, Stability, mechanical strength, uniformity etc. This debates the concept of selecting appropriate crystal for the use.

Same argument applies for the gel that is used to hold the crystals.

Also the arrangement of these crystals in the gel as specified is difficult.

The dimensions of these crystals must be very small so as to increase the memory density.

The electrodes or voltage sensors used in this process must be of smaller dimensions so as to maintain proper contact with crystal surfaces.

Erase operation is complicated since it involves restoring both charge and shape of the crystals.

\section{Advantages:}

The above mentioned approach improves the memory density by increasing the number of crystals

per unit length.

It also reduces the usage of electricity since we use electricity to erase the memory only.

It enhances the speed of read and write operations.

It also increases the life time of the memory device since crystals have longer life times when compared to other electronic devices.

It provides the scope of having flexible memory devices in the future instead of having rigid memory devices.

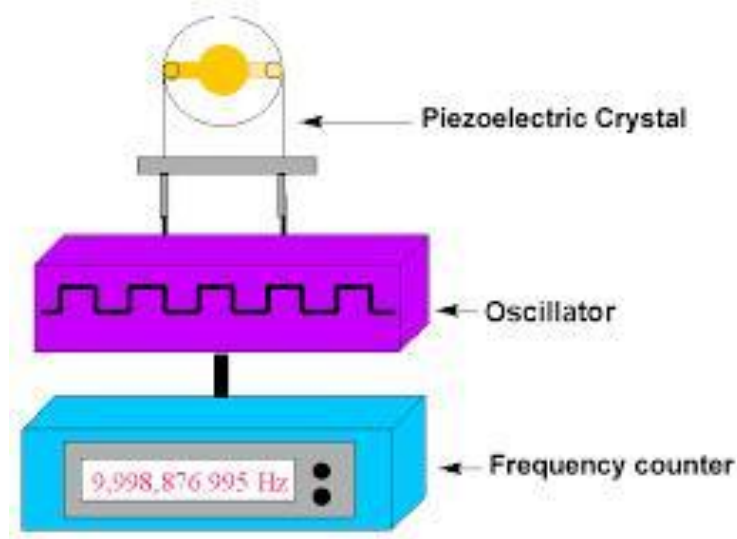

PiezoElectric crystal along with Oscillator

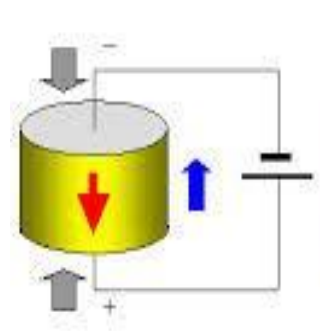

Shrink

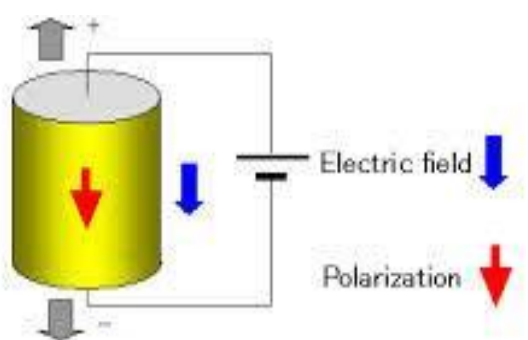

\section{Expand}

Working of peizo electricity

\section{PYROELCTRICITY}

Pyro electric crystals are crystals that generate electricity when heated. But the charge generated will be temporary only. Even though the heat is constantly applied, the crystal may initially get charged later again it may get discharged due to leakage current. Hence it is similar to DRAM which need to be refreshed periodically in order to retain data. Some natural minerals like Tourmaline is said to exhibit this property although efforts are made to construct artificial crystals that exhibit this property. This Property can be exploited to our use.

Arrangement of Crystals in gel: The arrangement of the crystals should be done in such a way that each crystal must be able to take the same amount of heat applied. It can be done by arranging the whole gel along with crystals in solid 3-dimensional objects like cylinder, cube etc. where crystals are placed on the surface as well as inside the solid shape in such a way that all are symmetrically located with respect to each other. This entire setup is enclosed with a skin or cover which is hard and strong. The skin must be a very good thermal insulator in order to avoid loss of heat in the crystals. It also provides the mechanical strength to the setup and retains shape.

Write Operation: The write operation is performed by exposing the selected crystals to certain temperature. This is done by placing a thermally conductive rod on the surface of crystal. Heat is provided to crystal through this rod. This rod must also act as voltage sensor in order to read the E.M.F generated in crystal. The crystals are placed in a gel which acts as an electric insulator. This helps 
crystals to retain their charge for a longer time. The crystals which are subjected to heat are considered to be storing bit ' 1 ', and the others have bit ' 0 ' in them. The crystals must be periodically read and refreshed by applying heat similar to DRAM cells.

Read Operation: This operation is performed by reading the E.M.F values in the crystals by voltage sensor. This voltage sensor reads bit ' 1 ' when there is E.M.F in the crystal or else it reads bit ' 0 '.

Erase Operation: This operation is performed by removing the E.M.F values in the crystals by the conducting rods. This brings back the crystals to their original state. The erase operation is quite easy in the crystals since they are prone to lose their induced E.M.F easily.

\section{Feasibility of the Suggested Approach}

In this approach we came to notice that the crystals used must be selected with extreme caution.

The selected crystals must have certain properties like pyro electricity, considerable rigidity and flexibility, Stability, mechanical strength, uniformity etc. This debates the concept of selecting appropriate crystal for the use.

Same argument applies for the gel that is used to hold the crystals.

Also the arrangement of these crystals in the gel as specified is difficult.

The dimensions of these crystals must be very small so as to increase the memory density.

The conducting metal rods or voltage sensors used in this process must be of smaller dimensions so as to maintain proper contact with crystal surfaces.

Loss of heat is difficult to be avoided.

\section{Advantages}

The above mentioned approach improves the memory density by increasing the number of crystals per unit length.

It also reduces the usage of electricity.

It enhances the speed of read, write and erase operations.

It also increases the life time of the memory device since crystals have longer life times when compared to other electronic devices.

It provides the scope of having flexible memory devices in the future instead of having rigid memory devices.

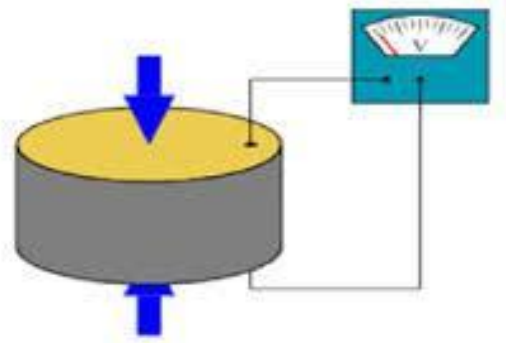

A pyro elctric crystal along with voltmeter

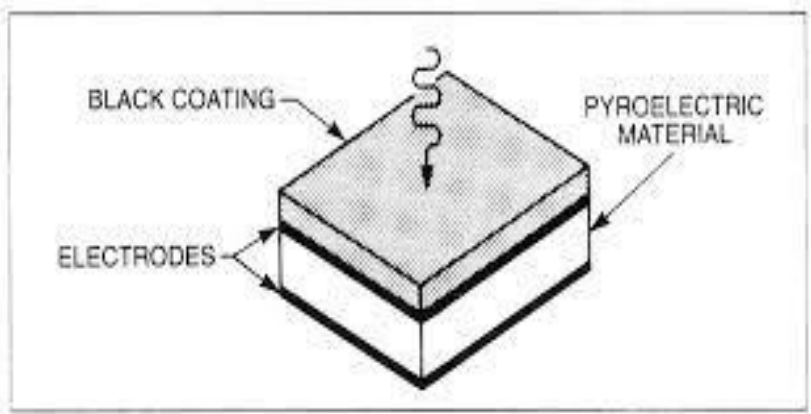

Fig. 6 Schematic of a pyroelectric detector.

\section{FERRO ELECTRICITY}

Ferro electricity is a property of certain materials that have a spontaneous electric polarization that can be reversed by application of an external electric field. It is found to be exhibited in certain minerals like Rochelle salt. Even in the absence of the external field they exhibit some nonzero polarization property. This property can be exploited to our use. When this crystal is subjected to external electric field in the opposite direction to that of internal electric field that is generated by dipoles the polarity of the crystals get reversed.

Arrangement of Crystals in gel: The arrangement of the crystals should be done in such a way that each crystal must be symmetrically located with respect to other. This entire setup is enclosed with a skin or cover which is hard and strong. This provides the mechanical strength to the setup and retains shape. Also the gel must be electrically conducting in nature so as to allow applied electric field to pass through it.

Write Operation: The write operation is performed by exposing the selected crystals to electric field in one direction. This direction is parallel to the direction of alignment of domains in the crystal there by increasing the strength of the electric field in the crystal. These crystals which are subjected to external electric field are considered to be storing bit ' 1 ' since the E.M.F across them is higher than that of the other crystals. Other crystals are considered to be storing bit ' 0 '.

So by setting a threshold value to the E.M.F across the crystal, we can say whether a crystal contains ' 0 ' or ' 1 '. 
Read Operation: This operation is performed by using a sense amplifier that reads the E.M.F across crystal. It compares this value with the threshold value. If it is greater than the threshold value it is considered as bit ' 1 '. If it is less than threshold it is considered as bit ' 0 '. These sense amplifiers or voltage sensors are placed such that they are capable of reading E.M.F across any crystals.

Erase Operation: This operation is performed by applying the external electric field in the opposite direction to that of inherent electric field of domains. The potential of this electric field applied must be greater than that of potential of electric field used for write operation. This will reduce the E.M.F across the crystals below the threshold value there by performing the erase operation.

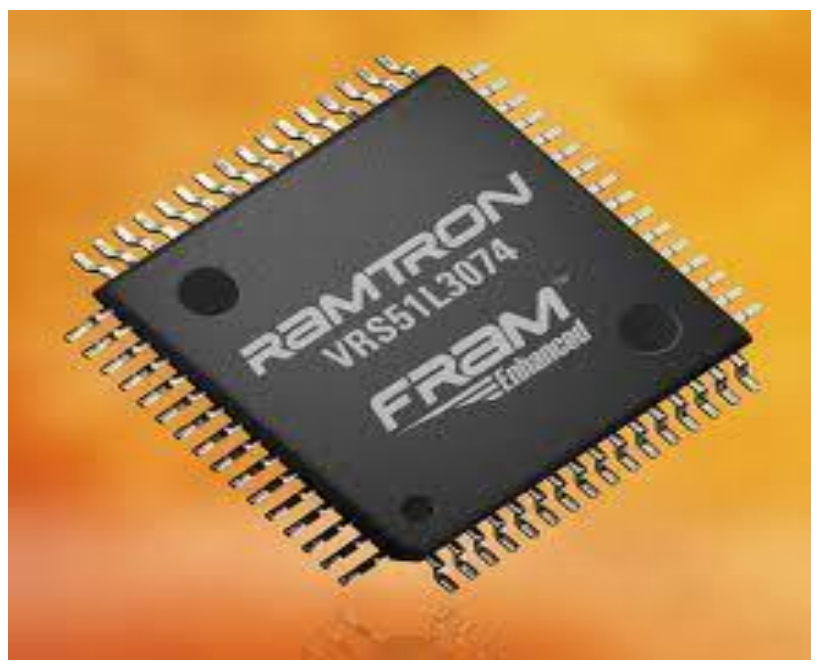

FerroElectric RAM Cell

\section{Feasibility of the Suggested Approach:}

In this approach we came to notice that the crystals used must be selected with extreme caution.

The selected crystals must exhibit ferro electricity property.

Same argument applies for the gel that is used to hold the crystals.

Also the arrangement of these crystals in the gel as specified is difficult.

The dimensions of these crystals must be very small so as to increase the memory density.

\section{Advantages}

The above mentioned approach improves the memory density by increasing the number of crystals per unit length.

It enhances the speed of read, write and erase operations.

It also increases the life time of the memory device since crystals have longer life times when compared to other electronic devices.
It provides the scope of having flexible memory devices in the future instead of having rigid memory devices.

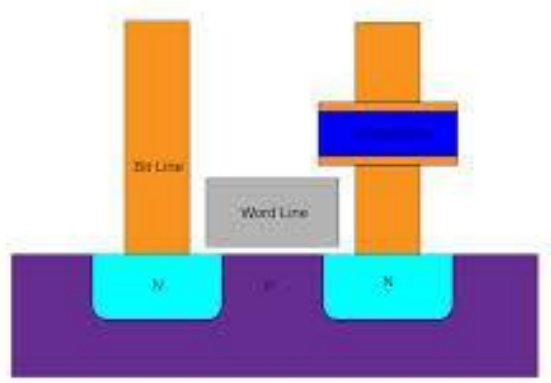

A FerroElectric RAM Working

\section{CONCLUSION}

By making use of any of the above mentioned methods we can make new a generation of memories which are flexible and compact. This research is already in progress and many big MNC s like IBM are into this. We hereby conclude our paper with a note to all readers:- This concept of using crystals and their properties for storing data is a budding concept and requires much more research. We hope we will publish our further research on each particular property mentioned above, for storing data in a more lucid manner.

\section{REFERENCES}

[1]. Wikipedia - Crystals, their properties, examples of crystals are collected from internet. 